\title{
Between Physics and Philosophy-Festschrift for Peter Mittelstaedt on His 80th Birthday
}

\author{
Paul Busch
}

Published online: 29 May 2010

(C) Springer Science+Business Media, LLC 2010

On 24 November 2009, Peter Mittelstaedt celebrated his eightieth birthday. The contributions by his colleagues and students collected here in three consecutive issues of Foundations of Physics have been specially written to pay tribute to his scientific achievements. The articles written for this Festschrift reflect the breadth of Peter Mittelstaedt's scientific oeuvre. Brigitte Falkenburg has contributed a review and appreciation of Peter Mittelstaedt's philosophical work. Also included is a survey of his research in theoretical physics and the foundations of quantum mechanics, which may serve as a guide to the enclosed bibliography of his publications.

"Between Physics and Philosophy" is the title of a two-day Symposion that was held in his honor at the University of Cologne in December 2009. Under this title we celebrated Peter Mittelstaedt as one of a small number of distinguished scholars who have successfully maintained the spirit and practice of physics as natural philosophy. Creatively crossing the boundary between physics and philosophy, he has helped to build bridges connecting these disciplines much to their mutual benefit.

A Festschrift Editorial entitled "Peter Mittelstaedt-Philosopher-Physicist", written for this journal in 1989 on the occasion of his sixtieth birthday, concluded with the sentence: "Foundations of physics, a delicate orchid among the firm and well-established branches of physics, will be a fortunate beneficiary indeed of his undiminished support and inspiration in the future." This sentence has come true indeed-Peter Mittelstaedt has continued to be highly productive, looking back now at an astounding time span of 55 years as an active researcher, always maintaining exemplary standards of intellectual rigor, clarity and depth in his scientific writing. The delicate orchid that Foundations of Quantum Mechanics used to be then has now grown into a strong and flourishing branch of fundamental physics, not least through

P. Busch (凶)

Department of Mathematics, University of York, York, UK

e-mail:pb516@york.ac.uk 
insistence, by Peter Mittelstaedt and like-minded academics of similar calibre, in the importance of clarifying the conceptual puzzles of quantum mechanics. Meanwhile this attitude has-surprisingly for some-borne rich fruit with the emergence of the new and rapidly developing field of quantum information science, which explores fundamental quantum phenomena as potentially powerful resources for the processing of information.

In a distinguished academic career starting in 1954 with a doctorate in theoretical physics under Werner Heisenberg, Peter Mittelstaedt has established himself as a leading scholar in areas as diverse as nuclear physics and quantum many-body theory, foundations of special and general relativity, quantum logic, foundations of quantum mechanics, and philosophy of science. A true polymath, he has also been a highly respected speaker, panelist and adviser in discussions on topics of wider interest, be it the interaction of science and society, ethical issues of biology, or the relationship between physics and theology.

Far from "retiring" in the transition from Professor to Emeritus Professor some 15 years ago, Peter Mittelstaedt has reaped a rich harvest from his earlier work on the foundations and philosophy of quantum mechanics, notably the Cologne brand of quantum logic, an operational reconstruction of Hilbert space quantum mechanics as a language for physical objects in which certain ontological prejudices of the language of classical physics have been eliminated. He has revisited the most notorious problems and paradoxes of quantum mechanics in the light of insights gained in the quantum logic approach. He has also undertaken to write integrative accounts of the underlying philosophical problems in the seminal book "The Interpretation of Quantum Mechanics and the Measurement Process" in 1997 and the monumental work "Laws of Nature" published in 2005 together with philosopher of science Paul Weingartner.

Furthermore, Peter Mittelstaedt has continued to share responsibilities for the organization of conferences, notably the 1993 Cologne sequel of the influential Joensuu Symposia on the Foundations of Modern Physics, the 1996 Biannual Meeting of the International Quantum Structures Association in Berlin, at the time of his presidency over this organization, a "Workshop on Superluminal (?) Velocities" in 1998, as well as a number of philosophical workshops.

Peter Mittelstaedt has not ceased to make himself available to young researchers in the foundations and philosophy of physics at international conferences, thus making his unique contribution to keeping alive the idea of Plato's symposion. Seeing him in conversations with young colleagues, it is evident that he thoroughly enjoys practicing the Socratic method in the search for scientific truth.

It remains for the writer to join all contributors to this Festschrift to congratulate Peter Mittelstaedt and to extend our very best wishes for many healthy and rewarding years to come. 